\title{
Clinical Profile of COVID-19 IIIness in Children-Experience from a Tertiary Care Hospital
}

\author{
Preeti Singh ${ }^{1} \cdot$ Karanvir Attri $^{1} \cdot$ Deonath Mahto ${ }^{1} \cdot$ Virendra Kumar $^{1} \oplus \cdot$ Dipti Kapoor $^{1} \cdot$ Anju Seth ${ }^{1} \cdot$ Varinder Singh $^{1}$. \\ Harish Pemde ${ }^{1} \cdot$ Praveen Kumar $^{1} \cdot$ Ravitanaya Sodani $^{1} \cdot$ Ankita Goel $^{1}$
}

Received: 26 December 2020 / Accepted: 24 May 2021 /Published online: 27 July 2021

(c) Dr. K C Chaudhuri Foundation 2021

\begin{abstract}
Objective To detail clinical profile and outcome in children infected with SARS-CoV-2.

Methods This retrospective study was undertaken at a tertiary care pediatric teaching hospital in Northern India. The data on clinical characteristics and outcome of children (<18 y) with COVID-19 illness from April 2020-October 2020 were reviewed and analyzed.

Results A total of 2919 children with suspected severe acute respiratory syndrome coronavirus 2 (SARS-CoV-2) illness were tested for novel COVID-19 virus in the flu emergency $(n=1744)$, severe acute respiratory infection (SARI) ward $(n=825)$, and non-COVID area $(n=350)$ of the hospital. $8.73 \%$ (255/2919) children tested positive for SARS-CoV-2 infection. Of the 255 positive cases, $144(56.47 \%$ ) were managed on an outpatient basis and 100 (59 boys) required admission in COVID ward. The mortality rate of patients with SARS-CoV-2 was $11.4 \%$ (29/255). Majority of children admitted with COVID-19 had severe to critical illness due to the presence of malnutrition and underlying comorbidities.

Conclusions Children of all age groups were susceptible to COVID-19 illness with a slight male preponderance. Amongst infected, two-third were asymptomatic or had mild symptoms that required outpatient management and home isolation. The adverse outcomes were more commonly seen in infants and children $>10 \mathrm{y}$ of age with malnutrition and comorbid illness.
\end{abstract}

Keywords Pandemic $\cdot$ COVID-19 illness $\cdot$ Comorbidities $\cdot$ Severe acute respiratory infection

\section{Introduction}

Novel Coronavirus disease 2019 (COVID-19) pandemic, caused by severe acute respiratory syndrome coronavirus 2 (SARS-CoV-2) is a global health and economic catastrophe of unprecedented magnitude. The disease affects all age groups and its spectrum varies from mild flu-like illness to severe pneumonia with complications like acute respiratory distress syndrome (ARDS), shock, multiorgan dysfunction, myocardial injury and acute kidney injury (AKI) [1]. Worldwide surveillance data have reported children typically to account for 13 percent of laboratory-confirmed cases.

Virendra Kumar

drvkumar1@gmail.com

Preeti Singh

drpreetisingh3@gmail.com

1 Department of Pediatrics, Lady Hardinge Medical College and Kalawati Saran Children's Hospital, New Delhi 110001, India
Children of all ages are susceptible to COVID-19 illness [2-4]. The disease in children is asymptomatic or mild in majority; however, critical illnesses are observed in small proportion [5, 6]. Despite the worldwide spread, the epidemiological and clinical patterns of the COVID-19 remain largely unclear, particularly among children. Since the virus is novel and there exists a lot of heterogeneity in the spectrum and severity of illness across age groups globally, it is pertinent to study and identify the clinical profile and outcome of these patients.

There have been a growing number of studies focused on COVID-19 illness but limited data are available on clinical profile and outcome on COVID-19 in children from India. Pediatric multisystem involvement has been reported as isolated case reports and case series [7, 8]. There is paucity of data on the impact of underlying diseases or pre-existing comorbidities on the severity of illness in children. Information on the characteristics of severely affected pediatric patients is also scarce. The present study aims to analyze the clinical features and outcome in children infected with SARS-CoV-2. 


\section{Material and Methods}

This retrospective study was undertaken at a tertiary care pediatric teaching hospital in Northern India. During the SARS-CoV-2 outbreak, a dedicated area for screening and triaging of patients with suspected COVID-19 illness was made operational, near the entrance gate of the hospital. This area was managed round the clock by a triage nurse, equipped with digital thermometer and finger pulse-oximeter. All children $(<18$ y of age) visiting the triage area with recent onset of fever and cough and/or fast breathing were referred to flu emergency for further evaluation. Flu emergency was created for initial management and sampling of suspected cases of COVID-19 in children. Besides, severe acute respiratory infection (SARI) ward and COVID ward were developed, where children with COVID-19-suspect and confirmed cases were admitted respectively. The criteria for admission for suspected COVID-19 illness included any of the following: respiratory distress, $\mathrm{SpO}_{2}$ on room air $<94 \%$, shock/poor peripheral perfusion, poor oral intake or lethargy, presence of seizures or encephalopathy [9]. Further, presence of comorbidities and/or age $<1 \mathrm{y}$ in COVID-suspect children were other indications for admission. The children with COVID19-suspected illness and requiring admission were shifted to SARI ward from flu emergency. The RT-PCR sample for novel COVID-19 virus was sent at admission in accordance with WHO guidelines [9]. Some children from non-COVID areas of the hospital were also screened for COVID-19 illness, if they developed suspicious symptoms after admission, came from containment zones/hotspots or had a history of contact with COVID-19 cases (undisclosed before). Based on the results of confirmatory RT-PCR and clinical assessment, hospital treatment or home isolation measures were instituted with contact tracing measures as applicable (in accordance with the local prevailing guidelines). The children with positive COVID-19 report and fulfilling the admission criteria were shifted to the COVID ward.

The cases were classified as mild, moderate, severe and critical as per the published guidelines [6]. Severe and critical cases requiring intensive monitoring, ventilation and organ support therapy were managed in the HDU/ICU of COVID ward. The remaining patients and those improving with care were managed in the step-down facility of the ward. The patients were subjected to investigations including complete blood count, serum biochemistry (liver and kidney function test, electrolytes), and chest radiograph. The coagulation profile, arterial blood gases, markers of inflammation including C-reactive protein, procalcitonin, serum ferritin, and CPK-MB were evaluated in those with severe COVID.

All the patients were managed as per the standard WHO protocol [9]. Paracetamol for fever control and intravenous or oral fluids to maintain adequate hydration and maintain fluid electrolyte balance was administered as required. Early initiation of nutrition was also considered in noncritically sick children. The patients developing respiratory distress or hypoxemia on room air $(<94 \%)$ were given oxygen support. In absence of specific guidelines for children, the authors followed the ICMR guidelines for treatment of COVID in adults with appropriate modifications [10]. Oseltamivir, azithromycin and hydroxychloroquine $(>12 \mathrm{y}$ ) were given to cases whenever indicated as per the prevailing guidelines. Empirical antibiotics were administered in cases with suspected sepsis or with shock and were managed as per the Surviving Sepsis Campaign Guidelines [11]. Children developing multisystem inflammatory syndrome (MIS-C) were managed with intravenous immunoglobulin (IVIG) and methylprednisolone as per the CDC guidelines for treatment of MIS-C [12, 13].

The case records of patients admitted in COVID ward were reviewed and the clinical, biochemical, radiological and management details recorded. The inclusion criteria were children up to $18 \mathrm{y}$ of age and with at least one positive RT-PCR test for novel COVID-19 virus. The patients who absconded or left against medical advice were excluded from the study. Details of each patient including demographic profile, clinical presentation and severity, comorbidities, treatment received, investigations (hematological, biochemical and radiological), course during hospital stay and outcome were recorded. Ethics approval for the study was obtained from the Institutional Ethics Committee.

Data was entered in EXCEL sheet. Descriptive statistics were used with continuous data represented as mean \pm SD and categorical data as counts and proportions.

\section{Results}

From April 2020 to October 2020, a total of 54,486 children were triaged at the screening centre depending upon their symptoms either to the flu emergency $(n=4184)$ or the general OPD/emergency $(n=50,302)$ areas (scheme of flow of patients in Fig. 1). There was a gradual rise in number of children attending hospital from April to June followed by a plateau in July-August and thereafter, a second peak was observed in September-October (Supplementary Fig. S1). A total of 2919 children with suspected SARS-CoV-2 illness were tested for novel COVID-19 virus in the flu emergency $(n=1744)$, SARI ward $(n=825)$ and non-COVID area $(n=350)$ of the hospital; $8.73 \%$ (255/2919) children tested positive for SARS-CoV-2 infection from different areas of the hospital (Table 1). Of 255 SARSCoV-2 positive cases, 144 (56.47\%) children were asymptomatic or had mild symptoms;managed on an outpatient basis and sent on home isolation. A total of 100 (59 boys) SARS-CoV-2 positive children required admission in COVID ward. The mortality rate of patients with SARS-CoV-2 was 11.4\% (29/255). 
Fig. 1 Scheme of triage and flow of patients admitted in different areas of the hospital during SARS CoV-2 pandemic $I P D$ Inpatient department; $O P D$ Outpatient department SARI Severe acute respiratory infection

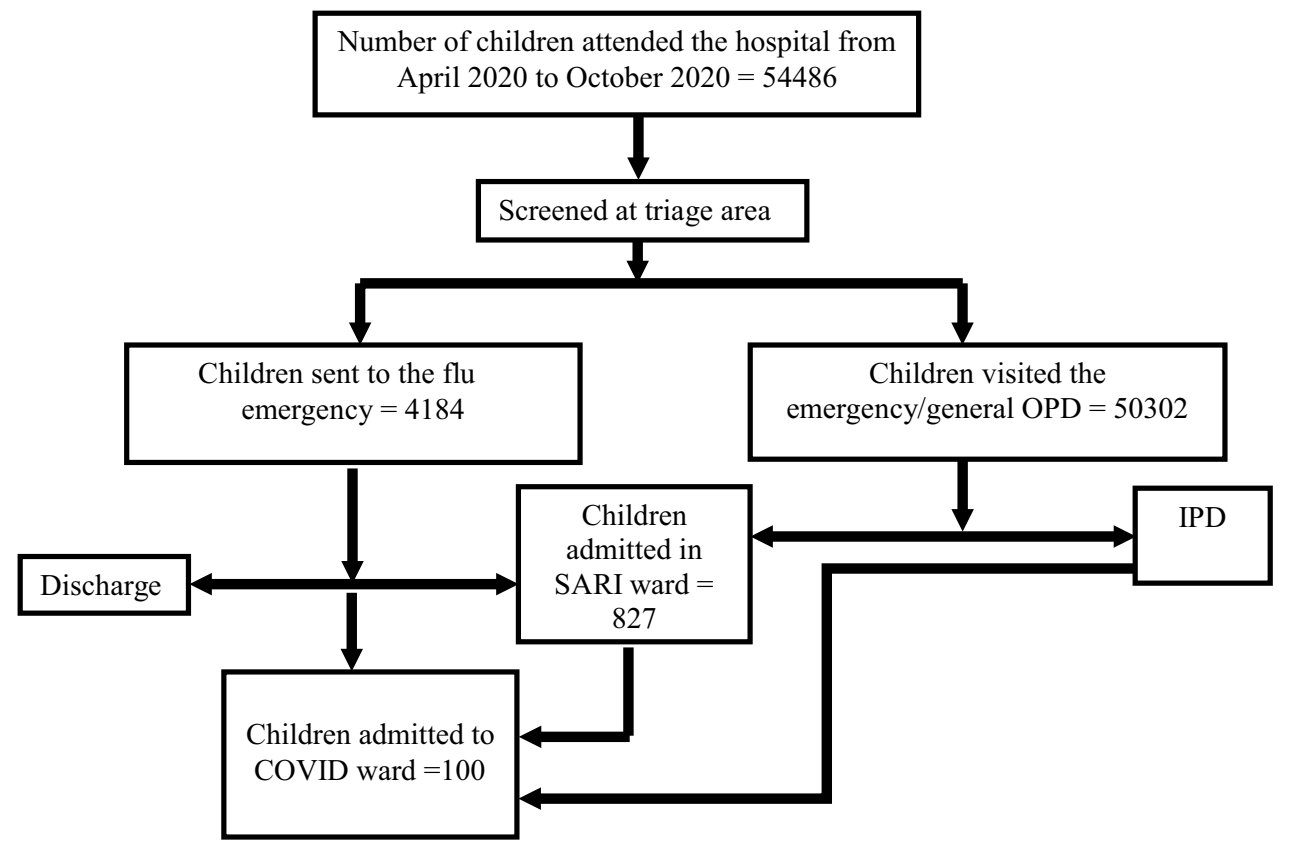

The median age of admitted children was $62.5 \mathrm{mo}$ (range, $10 \mathrm{~d}-18 \mathrm{y}$ ). The median time from onset of illness to diagnosis was $4 \mathrm{~d}$ (range $2-45 \mathrm{~d}$ ). The most common symptom observed amongst the admitted patients were fever (80\%), cough (35\%), fast breathing (47\%) and nausea/vomiting $(34 \%)$. Among the symptomatic patients moderate, severe and critical illness was seen in $27 \%, 42 \%$ and $31 \%$ patients, respectively. The proportion of children admitted in different age groups with their clinical profile is given in Table 2. More than half (59\%) of the patients admitted with SARSCoV-2 had underlying comorbid illness. Tuberculosis and hematological malignancy were the most commonly observed comorbidity in these children.

Amongst the children who died (29) with SARS-CoV-2 infection, 27 were admitted in COVID ward and 2 were brought dead; with male to female ratio of 1.45:1 The maximum proportion of deaths occurred in infants $(29.62 \%)$ and children $>10 \mathrm{y}(33.33 \%)$ in the absence and presence of comorbid illness respectively. The most frequent complication observed in the admitted children with SARS-CoV-2 was acute respiratory distress syndrome $(18 \%)$ followed by acute kidney injury (AKI) (16\%), encephalopathy (15\%) and myocarditis (9\%) (Table 3). Multisystem inflammatory syndrome in children (MIS-C) was present in 9\% patients. Three patients required renal replacement therapy in lieu of AKI not responding to medical management.

Among children on respiratory support, 54\% required oxygen inhalation, $11 \%$ were put on noninvasive ventilation (HFNC/CPAP) and $35 \%$ needed mechanical ventilation. The mean (SD) hospital stay was of 8.95 (7.77) d (median-7

Table 1 Distribution of patients with SARS-CoV-2 infection from different zones of the hospital

\begin{tabular}{|c|c|c|c|}
\hline & $\begin{array}{l}\text { Non-COVID area* } \\
(\%)\end{array}$ & $\begin{array}{l}\text { Flu emergency } \\
(\%)\end{array}$ & $\begin{array}{l}\text { SARI ward } \\
(\%)\end{array}$ \\
\hline $\begin{array}{l}\text { Total cases screened for SARS-CoV-2 } \\
(n=2919)\end{array}$ & $350(12 \%)$ & $1744(59.7 \%)$ & $825(28.3 \%)$ \\
\hline Brought dead $^{\mathrm{a}}(n=2)$ in different zones & 1 & 1 & - \\
\hline $\begin{array}{l}\text { Total SARS-CoV-2 positive cases } \\
(n=255)\end{array}$ & $60(23.5 \%)$ & $148(58 \%)$ & $47(18.5 \%)$ \\
\hline $\begin{array}{l}\text { Children admitted with SARS CoV-2 from different zones } \\
(n=100)\end{array}$ & $40(40 \%)$ & $20(20 \%)$ & $40(40 \%)$ \\
\hline $\begin{array}{l}\text { Managed on an outpatient basis and sent on home isolation in different zones } \\
(n=144)\end{array}$ & $17(11.8 \%)$ & $122(84.7 \%)$ & $5(3.5 \%)$ \\
\hline $\begin{array}{l}\text { Transfer to other hospitals from different zones } \\
(n=10)\end{array}$ & $3(30 \%)$ & $5(50 \%)$ & $2(20 \%)$ \\
\hline
\end{tabular}

*Non-COVID area includes emergency, Non-COVID ICU, Inpatient wards

${ }^{\mathrm{a}} \mathrm{COVID}-19$ positive on postmortem examination 
Table 2 Clinical characteristics of children admitted with COVID-19 illness

\begin{tabular}{|c|c|c|c|}
\hline \multicolumn{2}{|l|}{ Characteristic } & \multicolumn{2}{|l|}{ Number $(N=100)$} \\
\hline \multirow{2}{*}{\multicolumn{2}{|c|}{$\begin{array}{l}\text { Age in months, median (IQR) } \\
\text { Mean } \pm \text { SD }\end{array}$}} & \multicolumn{2}{|l|}{$62.5(16.25,120)$} \\
\hline & & $72.73 \pm 57.68$ & \\
\hline \multicolumn{2}{|l|}{ Age group (y) } & \multicolumn{2}{|l|}{$n(\%)$} \\
\hline \multicolumn{2}{|l|}{$<1$} & \multicolumn{2}{|l|}{$24(24)$} \\
\hline \multicolumn{2}{|l|}{$1-5$} & \multicolumn{2}{|l|}{$26(26)$} \\
\hline \multicolumn{2}{|l|}{$5-10$} & \multicolumn{2}{|l|}{$25(25)$} \\
\hline \multicolumn{2}{|l|}{$>10$} & \multicolumn{2}{|l|}{$25(25)$} \\
\hline \multicolumn{2}{|l|}{ Sex (M:F) } & \multicolumn{2}{|l|}{1.44} \\
\hline \multicolumn{4}{|l|}{$\begin{array}{l}\text { Anthropometry } \\
\text { (SD score) Median (IQR)/Mean } \pm \text { SD }\end{array}$} \\
\hline \multicolumn{2}{|l|}{ Weight/Age } & \multicolumn{2}{|c|}{$-1.51(-2.61,-0.61) /-1.79 \pm 1.89$} \\
\hline \multicolumn{2}{|l|}{ Weight/Length $(<5$ y) } & \multicolumn{2}{|c|}{$-1.72(-2.55,-0.73) /-1.85 \pm 1.54$} \\
\hline \multirow[t]{2}{*}{ BMI/Age (>2 y) } & & \multicolumn{2}{|c|}{$-1.45(-2.11,-0.55) /-1.65 \pm 1.45$} \\
\hline & SD score & \multicolumn{2}{|l|}{$n(\%)$} \\
\hline Weight/age & $\begin{array}{l}<-2 \\
<-3\end{array}$ & $\begin{array}{l}38(38) \\
18(18)\end{array}$ & \\
\hline Weight/length & $\begin{array}{l}<-2 \\
<-3\end{array}$ & $\begin{array}{l}31(31) \\
22(22)\end{array}$ & \\
\hline BMI/Age & $\begin{array}{l}<-2 \\
<-3\end{array}$ & $\begin{array}{l}29(29) \\
16(16)\end{array}$ & \\
\hline Clinical Features & & $n(\%)$ & $\begin{array}{l}\text { Duration of symptoms (d) } \\
\text { Median (IQR) / Mean } \pm \text { SD }\end{array}$ \\
\hline Fever & & $80(80)$ & $4(2,7) / 10.34 \pm 28.05$ \\
\hline Cough & & $35(35)$ & $3(2,7) / 6.46 \pm 7.47$ \\
\hline Fast breathing & & $47(47)$ & $2(1,5) / 3.41 \pm 3.36$ \\
\hline Pneumonia & & $46(46)$ & - \\
\hline Headache & & $11(11)$ & $4(2,5) / 3.64 \pm 1.96$ \\
\hline Myalgia & & $11(11)$ & - \\
\hline Nausea and vomiting & & $34(34)$ & $2(1,4) / 3.26 \pm 3.40$ \\
\hline Diarrhea and abdominal pain & & $27(27)$ & $3(2,5) / 4.41 \pm 4.19$ \\
\hline Altered sensorium & & $15(15)$ & - \\
\hline Rash & & $9(9)$ & $2(1,5) / 3.56 \pm 3.16$ \\
\hline Hypoxemia ( $\mathrm{SpO}_{2}<94 \%$ ) at admission & & $42(42)$ & - \\
\hline Severity of illness & & $n(\%)$ & \\
\hline Moderate & & $27(27)$ & \\
\hline Severe & & $42(42)$ & \\
\hline Critical & & $31(31)$ & \\
\hline Comorbidities & & $\begin{array}{l}n(\%) \\
59(59)\end{array}$ & $\begin{array}{l}\text { Deaths secondary to COVID- } \\
\text { related illness }(11 / 59)\end{array}$ \\
\hline Tuberculosis & & $20(20)$ & 4 \\
\hline Liver abscess & & $6(6)$ & - \\
\hline Disseminated staphylococcus sepsis & & $4(4)$ & 1 \\
\hline Aplastic anemia/ALL/AML & & $15(15)$ & 3 \\
\hline Dengue & & $4(4)$ & 1 \\
\hline Nephrotic syndrome & & $2(2)$ & - \\
\hline SLE & & $1(1)$ & 1 \\
\hline SOJIA & & $1(1)$ & 1 \\
\hline Type 1 DM & & $1(1)$ & - \\
\hline *Others & & $5(5)$ & - \\
\hline History of contact with SARS-CoV-2 & & 12 & \\
\hline Time from onset of illness to hospitalisati & & & \\
\hline Median (IQR) /Mean \pm SD & & $4(2,7) / 6.42 \pm 6.81$ & \\
\hline
\end{tabular}

$I Q R$ Interquartile range, $S D$ Standard deviation

* Others include congenital conditions like CCAM, surgical conditions like abdominal cystic mass, glomerulonephritis with hypertension, and Tay-Sachs disease 
Table 3 Clinical course and outcome of children with SARS-CoV-2 infection admitted in COVID ward

\begin{tabular}{lll}
\hline Characteristic $(N=100)$ & $n$ & $\%$ \\
Complications & & \\
- Acute respiratory distress syndrome & 18 & 18 \\
- Encephalopathy & 15 & 9 \\
- Myocarditis & 9 & 19 \\
- Transaminitis & 19 & 16 \\
- Acute kidney injury & 16 & 35 \\
Shock requiring vasoactive support & 35 & \\
Maximal respiratory support & & 54 \\
Oxygen & 54 & 11 \\
NIV (HFNC, CPAP) & 11 & 35 \\
Mechanical ventilation & 35 & 9 \\
MISC & 9 & 3 \\
Renal replacement therapy & 3 & \\
Length of hospital stay (d) & & 70 \\
Mean \pm SD/Median (IQR) & $8.95 \pm 7.77 / 7(4,13)$ \\
Outcome & & 27 \\
Discharge & 70 & 3 \\
Death & 27 & 3 \\
Transfer to other hospitals & 37 & \\
\hline
\end{tabular}

$C P A P$ Continuous positive airway pressure airway pressure; $H F N C$ High flow nasal cannula; IQR Interquartile range; MISC Multisystem inflammatory syndrome in children; NIV Noninvasive ventilation

$\mathrm{d}$ : range, $1-30 \mathrm{~d})$. The baseline laboratory parameters of the admitted patients with SARS-CoV-2 infection are outlined in Table 4. Two-third of the children had abnormal blood counts; leucocytosis $(55 \%)$ was more commonly reported than leukopenia (12\%) and lymphopenia (28\%). Radiologically confirmed pneumonia was observed in $44 \%$ (40/66) of children. Negative RT-PCR results were obtained after a mean (SD) time of 12 (2) d (50\% by 10 th day, $75 \%$ by the 14 th day, $94 \%$ by the 21 st day), while one case remained positive until the 28th day.

\section{Discussion}

Addressing COVID-19 pandemic was challenging in a resource constrained developing country. Limited health care facilities and large vulnerable population, many of whom were already burdened with comorbid conditions posed challenges on every dimension. This retrospective study highlights the clinical profile of children with SARSCoV-2 infection from a tertiary care pediatric teaching hospital in northern India. Most of the children were either asymptomatic contacts or had mild flu-like symptoms. The most common presentation was fever followed by cough and fast breathing, but a substantial proportion had gastrointestinal symptoms as well. A significant proportion of children required admission due to associated comorbidities and severe illness. The authors also observed a higher rate of complications and mortality in the present study, presumably attributed to referral bias to the authors' center (higher proportion of critically ill children) and the presence of comorbidities. The adverse outcomes were more commonly seen in infants and children more than $10 \mathrm{y}$ of age.

The trend of total footfall of children attending the hospital was proportional to the lockdown and the unlock phase of the country (April to October 2020). However, the secondary peak in number of cases in September-October was associated with the return of the migrant population, overcrowding and the seasonal influence of surge in vector borne diseases (dengue, malaria) observed in the city. The mean age and sex distribution of children admitted with SARS-CoV-2 infection in this study was comparable with the studies published elsewhere [6]. Children of all age groups were susceptible to COVID-19 infection as reported elsewhere [14, 15]. Although the clinical features of COVID-19 illness in children are diverse, still fever and cough were the most common symptoms reported in this cohort. The spectrum of clinical features reported in other published studies are heterogeneous depending on the setting and the cohort analyzed $[5,16]$. Unlike the studies on disease severity from different settings, more than two third of the admitted cases in this study had severe to critical illness. Similar to the results reported by Dong et al. [6], infants were more vulnerable to SARS-CoV-2 infection and had more severe disease. However, the data on relationship of disease severity and gender are limited in children.

A striking observation of this study was that a high proportion of children with SARS-CoV-2 infection required admission in view of their underlying comorbid illness. Comorbidities among children with SARS-CoV-2 have been reported in studies from China [15], India (61\%) [17] and US/Canada (83\%) [18]. Around 50\% of children admitted with COVID-19 illness in this cohort had undernutrition and thinness that further compounded the severity of illness and morbidity. No comprehensive data are yet available on the impact of SARS-CoV-2 on children who are well and in those with an underlying illness; but it is reasonable to consider that the latter might be at an increased risk of severe disease. The mortality rate observed in this study is much higher than that reported globally in both children and adults $[19,20]$. The proposed reasons for this observation are referral bias and admissions of the most critically ill children, higher transportation time due to limited means during lockdown, comorbidities, malnutrition and infections prevalent in the authors' setting.

Contrary to published literature on laboratory parameters [18-23], a higher proportion of children in this study had anemia, leucocytosis, thrombocytopenia and elevated D-dimers. It can be attributed to higher rate comorbid illness (leukemia), secondary bacterial infections and sepsis in the authors' patients. However, the proportion of children with 
Table 4 Laboratory parameters in children admitted with SARS-CoV-2 infection

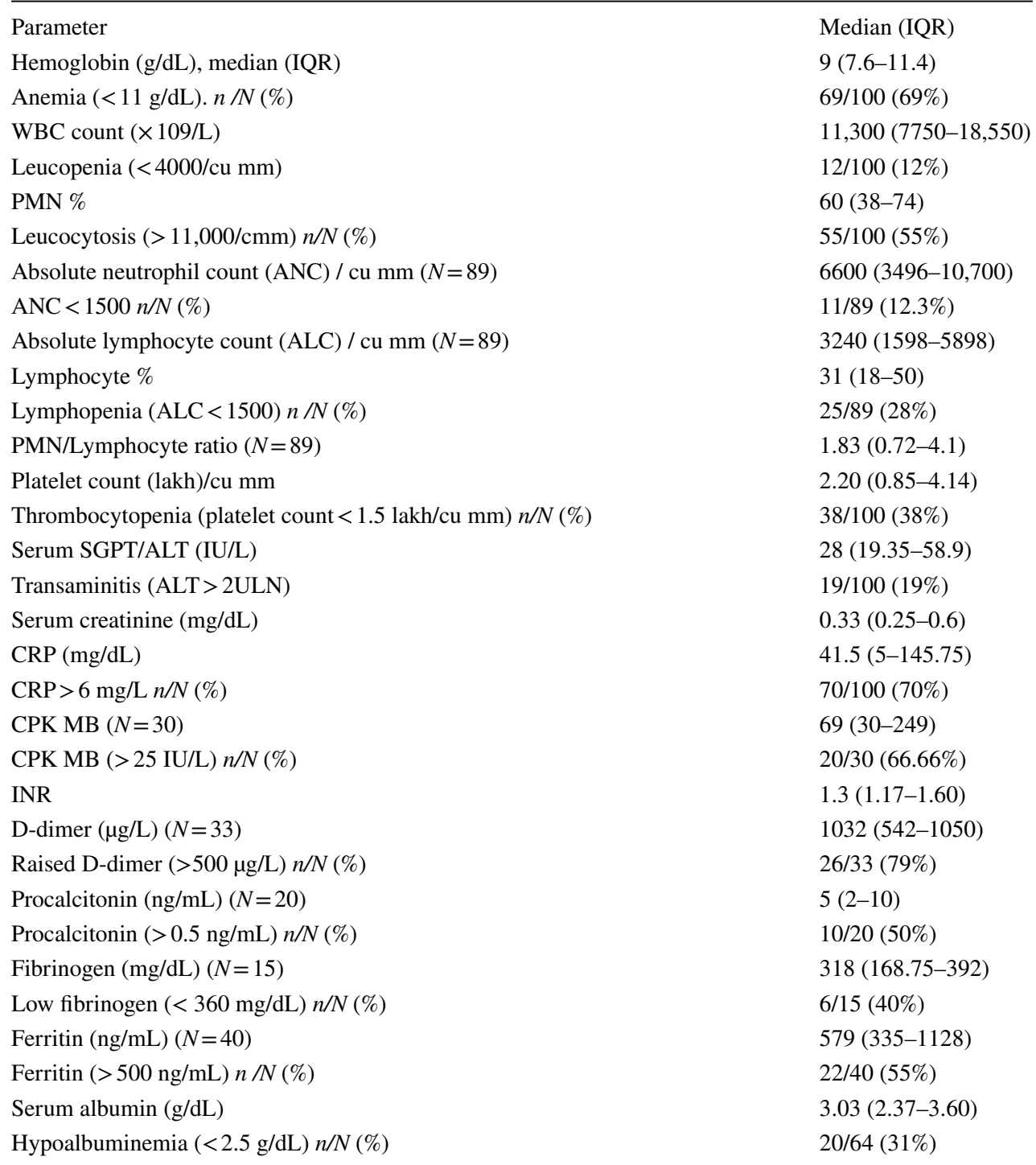

$I Q R$ Interquartile range; $N$ Total number of children for which the data were available; $n$ Number of children with a specific laboratory parameter hypoalbuminemia, high serum ferritin levels and elevated CPK-MB levels were similar to that reported in previous studies [21-23]. Most of the admitted children had severe and critical illness that resulted in higher proportion of them with these biomarkers of inflammation.

In contrast to the available literature on ventilation in SARS-CoV-2 [24], a higher proportion of children in the present study required oxygen and ventilatory support. The presence of comorbidities, like pulmonary tuberculosis, tubercular meningitis, disseminated staphylococcus infections and liver abscess accounted for increased need of respiratory support in the children included in the study. Children with MIS-C seen in this study was similar to previous reports from India [25] and other parts of the world [26, 27].

The study has its share of limitations of retrospective design and lack of long term follow up of comorbidities after discharge. In spite of these shortcomings, this study provides preliminary data on clinical characteristics and outcomes of COVID-19 in children from Northern India.

\section{Conclusion}

Children of all age groups were susceptible to COVID-19 illness with a slight male preponderance. Amongst infected, two-third were asymptomatic or had mild symptoms that required outpatient management and home isolation. Though fever and other respiratory symptoms make up the commonest clinical presentation, many may present with gastrointestinal symptoms. Majority of children admitted with COVID-19 had severe to critical illness due to the presence of malnutrition and underlying comorbidities. 
Supplementary Information The online version contains supplementary material available at https://doi.org/10.1007/s12098-021-03822-5.

Acknowledgements The authors thank all healthcare staff involved in the management of COVID-19 pandemic

Authors' Contribution PS: Execution of the study, data analysis, and writing the manuscript; KA: Data compilation, data analysis, and writing the manuscript; DM: Data analysis and writing the manuscript. VK: Planning, execution, data analysis, and writing the manuscript; DK: Data analysis and writing the manuscript; AS: Data analysis and writing the manuscript; VS: Data analysis and writing the manuscript; HP: Data analysis and writing the manuscript; PK: Data analysis and writing the manuscript; RS: Data analysis and writing the manuscript; AG: Data analysis and writing the manuscript. VK is the guarantor for this paper.

\section{Availability of Data and Material Yes.}

\section{Declarations}

Ethical Clearance The study was approved by the Institutional Ethics Committee for Human Research, Lady. Hardinge Medical College and associated hospitals, New Delhi, India.

\section{Conflict of Interest None.}

\section{References}

1. Wang D, Hu B, Hu C, et al. Clinical characteristics of 138 hospitalized patients with 2019 novel coronavirus-infected pneumonia in Wuhan. China JAMA. 2020;323:1061-9.

2. Gupta N, Praharaj I, Bhatnagar T, et al. Severe acute respiratory illness surveillance for coronavirus disease 2019, India, 2020. Indian J Med Res. 2020;151:236-40.

3. Stokes EK, Zambrano LD, Anderson KN, et al. Coronavirus Disease 2019 case surveillance - United States, January 22-May 30, 2020. MMWR Morb Mortal Wkly Rep. 2020;69:759-65.

4. Wu Z, McGoogan JM. Characteristics of and important lessons from the coronavirus disease 2019 (COVID-19) outbreak in China: summary of a report of 72314 cases from the chinese center for disease control and prevention. JAMA. 2020;323:1239-42.

5. Han MS, Choi EH, Chang SH, et al. Clinical Characteristics and Viral RNA Detection in Children with coronavirus disease 2019 in the Republic of Korea. JAMA Pediatr. 2021;175:73-80.

6. Dong Y, Mo X, Hu Y, et al. Epidemiology of COVID-19 among children in China. Pediatrics. 2020;145:e20200702.

7. Balasubramanian S, Nagendran TM, Ramachandran B, Ramanan AV. Hyper-inflammatory syndrome in a child with covid-19 treated successfully with intravenous immunoglobulin and tocilizumab. Indian Pediatr. 2020;57:681-3.

8. Dhanalakshmi K, Venkataraman A, Balasubramanian S, et al. Epidemiological and clinical profile of pediatric inflammatory multisystem syndrome - temporally associated with SARS-CoV-2 (PIMS-TS) in Indian children. Indian Pediatr. 2020;57:1010-4.

9. Clinical management of severe acute respiratory infection when COVID19 is suspected. Available at: https://www.who.int/publications-detail/ clinical-management-of-severe-acuterespiratory-infection-whennovel-coronavirus-(ncov)-infection-issuspected. Accessed on 31 Oct 2020.

10. Revised Guidelines on Clinical Management of COVID - 19 Government of India. Ministry of Health \& Family Welfare Directorate General of Health Services (EMR Division).

11. Weiss SL, Peters MJ, Alhazzani W, et al. Surviving Sepsis campaign international guidelines for the management of septic shock and sepsis-associated organ dysfunction in children. PediatrCrit Care Med. 2020;21:e52-106.

12. Nakra NA, Blumberg DA, Herrera-Guerra A, Lakshminrusimha S. Multi-system inflammatory syndrome in children (MIS-C) following SARS-CoV-2 infection: review of clinical presentation, hypothetical pathogenesis, and proposed management. Children (Basel). 2020;7:69.

13. Information for healthcare providers about multisystem inflammatory syndrome in children (MIS-C). In: Centers for Disease Control and Prevention. Available at: https://www.cdc.gov/mis-c/ hcp/. Accessed on 12 Aug 2020.

14. Choi S-H, Kim HW, Kang J-M, Kim DH, Cho EY. Epidemiology and clinical features of coronavirus disease, in children. Clin Exp Pediatr. 2019;2020(63):125-32.

15. Shekerdemian LS, Mahmood NR, Wolfe KK, et al. Characteristics and outcomes of children with coronavirus disease 2019 (COVID-19) infection admitted to US and Canadian pediatric intensive care units. JAMA Pediatr. 2020;174:868-73.

16. Liguoro I, Pilotto C, Bonanni M, et al. SARS-COV-2 infection in children and newborns: a systematic review. Eur J Pediatr. 2020;179:1029-46.

17. Banerjee S, Guha A, Das A, Nandi M, Mondal R. A Preliminary Report of COVID-19 in Children in India. Indian Pediatr. 2020;57:963-4.

18. Lu X, Zhang L, Du H, et al. SARS-CoV-2 infection in children. N Engl J Med. 2020;382:1663-5.

19. Meena J, Yadav J, Saini L, Yadav A, Kumar J. Clinical features and outcome of SARS-CoV-2 infection in children: a systematic review and meta-analysis. Indian Pediatr. 2020;57:820-6.

20. Hoang A, Chorath K, Moreira A, et al. COVID-19 in 7780 pediatric patients: a systematic review. Eclinical Medicine. 2020;24:100433.

21. Qiu H, Wu J, Hong L, Luo Y, Song Q, Chen D. Clinical and epidemiological features of 36 children with coronavirus disease 2019 (COVID-19) in Zhejiang, China: an observational cohort study. Lancet Infect Dis. 2020;20:689-96.

22. Chen N, Zhou M, Dong X, et al. Epidemiological and clinical characteristics of 99 cases of 2019 novel coronavirus pneumonia in Wuhan, China: a descriptive study. Lancet. 2020;395:507-13.

23. Rodriguez-Morales AJ, Cardona-Ospina JA, Gutiérrez-Ocampo E, et al. Clinical, laboratory and imaging features of COVID-19: a systematic review and meta-analysis. Travel Med Infect Dis. 2020;34:101623.

24. Patel NA. Pediatric COVID-19: a systematic review of the literature. Am J Otolaryngol. 2020;41:102573.

25. Acharyya BC, Acharyya S, Das D. Novel coronavirus, mimicking kawasaki disease in an infant. Indian Pediatr. 2020;57:753-4.

26. Verdoni L, Mazza A, Gervasoni A, et al. An outbreak of severe kawasaki-like disease at the Italian epicentre of the SARS-CoV-2 epidemic: an observational cohort study. Lancet. 2020;395:1771-8.

27. Viner RM, Whittaker E. Kawasaki-like disease: emerging complication during the COVID-19 pandemic. Lancet. 2020;395:1741-3.

Publisher's Note Springer Nature remains neutral with regard to jurisdictional claims in published maps and institutional affiliations. 